\title{
PERDA DE PESO E O COMPRIMENTO DO INTESTINO DELGADO NA CIRURGIA DE FOBI-CAPELLA: HÁ RELAÇÃO?
}

\author{
WEIGHT LOSS AND THE LENGTH OF THE SMALL INTESTINE \\ IN THE FOBI-CAPELLA SURGERY: IS THERE A RELATIONSHIP? \\ Oona Tomiê DARONCH ${ }^{1 \oplus}$, Hugo Genki Kagawa AKAHANE ${ }^{\oplus}$, Solange dos Anjos Cravo BETTINI ${ }^{\oplus}$
}

\begin{abstract}
RESUMO - RACIONAL: Os fatores relacionados à perda de peso nos pacientes obesos submetidos à cirurgia bariátrica sempre foram exaustivamente estudados na tentativa de propor a melhor técnica cirúrgica com maior perda de peso e resolução as comorbidades à longo prazo. Os pacientes apresentam variações anatômicas no que tange o comprimento do intestino delgado. Há estudos que demonstram alterações de peso nos pacientes que apresentam diferentes comprimentos das alças intestinais na técnica do by-pass em Y de Roux. O presente trabalho realizou um estudo entre a influência do IMC, a perda de peso e o comprimento da alça comum nos resultados cirúrgicos à longo prazo. MÉTODOS: Estudo transversal descritivo pela análise retrospectiva de 112 prontuários de pacientes submetidos à cirurgia bariátrica aberta pela técnica do bypass gástrico no Hospital de Clínicas -UFPR. Os dados foram correlacionados em programas estatísticos para este fim. RESULTADOS: Dos 112 pacientes, 83,03\% eram do sexo feminino, média de idade de 41,52 anos. O comprimento médio do intestino delgado total dos pacientes foi de 5,02 metros. Houve uma relação diretamente proporcional entre o comprimento do intestino delgado e a perda de peso $(p=0,0428)$. CONCLUSÃO: Há uma ampla gama de variáveis relacionadas à perda de peso nos pacientes submetidos à cirurgia bariátrica, tais como a técnica utilizada, o comprimento das alças no by-pass gástrico em $Y$ de Roux e a rotina de acompanhamento nutricional e físico do paciente. É importante considerar os detalhes técnicos do procedimento cirúrgico, e verificar a perda de peso avaliando-se o paciente como um todo e outras variáveis.

DESCRITORES: Cirurgia Bariátrica. Anastomose em - Y de Roux. Perda de Peso.
\end{abstract}

ABSTRACT - BACKGROUND: Factors related to weight loss in obese patients undergoing bariatric surgery have always been exhaustively studied in an attempt to propose the best surgical technique with greater weight loss and long-term resolution of comorbidities. Patients present anatomical variations regarding the length of the small intestine. Some studies demonstrate weight changes in patients with different lengths of the intestinal loops in the Roux-en-Y bypass technique. The present work carried out a study on the influence of body mass index, weight loss, and common loop length on long-term surgical outcomes. METHODS: This is a descriptive cross-sectional study by retrospective analysis of 112 medical records of patients undergoing open bariatric surgery using the gastric bypass technique at University Hospital - UFPR. The data were correlated in statistical programs for this purpose. RESULTS: Out of 112 patients, $83.03 \%$ were women, with mean age of 41.52 years. The mean length of the total small bowel of the patients was $5.02 \mathrm{~m}$. There was a directly proportional relationship between the length of the small intestine and weight loss $(p=0.0428)$ CONCLUSION: There is a wide range of variables related to weight loss in patients undergoing bariatric surgery, such as the technique used, the length of the loops in the Roux-en-Y gastric bypass, and the routine of nutritional and physical monitoring of the patient. It is important to assess the technical details of the surgical procedure and to verify the weight loss by evaluating integrally the patient and other variables.

HEADINGS: Bariatric Surgery. Anastomosis, Roux-en-Y. Weight Loss.

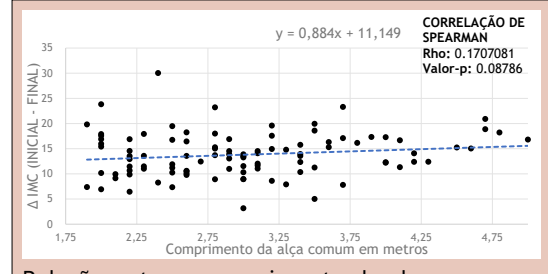

Relação entre o comprimento da alça comum e a perda de peso, avaliada pela diferença entre os IMC final e inicial $(p=0,08786)$

Mensagem central
A cirurgia bariátrica é uma das principais
indicações para perda de peso em pacientes
que não conseguem obter sucesso com o
tratamento clínico e mudança nos hábitos
de vida. Diversas variáveis influenciam no
emagrecimento, pois sabe-se que os diferentes
indivíduos com IMC semelhantes, mesmo
quando submetidos à mesma técnica operatória,
apresentam diferentes resultados na perda de
peso à longo prazo.

\section{Perspectivas}

Nesta pesquisa foi possível verificar uma relação diretamente proporcional entre o comprimento do intestino delgado e a perda de peso. Portanto, é importante avaliar não apenas detalhes técnicos do procedimento cirúrgico, mas verificar a perda de peso avaliando-se o paciente como um todo e as outras variáveis presentes.
Correspondência:

Oona Tomiê Daronch.

Email: oona.daronch@yahoo.com.br oona.daronch@gmail.com
Fonte de financiamento: Não

Conflito de interesses: Não

Recebido: 11/07/2021

Aceito: 30/09/2021 


\section{INTRODUÇÃO}

A cirurgia bariátrica tem se mostrado uma das principais estratégias de perda de peso em pacientes que não conseguem obter sucesso com o tratamento clínico e mudança nos hábitos de vida ${ }^{5}$. Existem diversas variáveis que influenciam no emagrecimento, pois sabe-se que os diferentes indivíduos com IMC semelhantes, mesmo quando submetidos à mesma técnica de cirurgia bariátrica, apresentam diferentes resultados na perda de peso à longo prazo ${ }^{5}$.

A indicação para cirurgia bariátrica atual em nosso meio são os pacientes que apresentam IMC $>35 \mathrm{~kg} / \mathrm{m}^{2}$ ou IMC $>40 \mathrm{~kg} / \mathrm{m}^{2}$ em pacientes com comorbidades. Os fatores relacionados à perda de peso nos pacientes obesos submetidos à cirurgia bariátrica sempre foram exaustivamente estudados na tentativa de propor a melhor técnica cirúrgica com maior perda de peso e resolução das comorbidades à longo prazo. Sabe-que que os pacientes apresentam variações anatômicas no que tange o comprimento do intestino delgado, o qual tem seu comprimento variado de $3,36 \mathrm{~m}$ a 7,64 m, apresenta diâmetro de aproximadamente $4 \mathrm{~cm}$, representando aproximadamente uma área de $250 \mathrm{~m}^{2}$ 2,6,7.

Apesar de serem intensamente estudado os fatores individuais metabólicos, genéticos e hábitos de vida, percebeu-se que os pacientes apresentam diferentes comprimentos de intestino delgado, e que esse fato poderia apresentar associação com a perda de peso, tendo em vista que o delgado é primariamente responsável pela absorção dos diferentes nutrientes. Além disso, na cirurgia de Fobi-Capella, uma das técnicas mais utilizadas em nosso meio, diferentes cirurgiões utilizam comprimentos distintos de comprimento de alça alimentar e biliopancreática, suscitando-se a hipótese de que esses comprimentos poderiam influenciar na perda de peso $3,10,12$

Tendo em vista esses diferentes fatores que podem influenciar na perda de peso e melhora das principais comorbidades dos pacientes à longo prazo, o objetivo deste trabalho busca elucidar os tópicos que precisam ser destacados na realização de cirurgia bariátrica nos diferentes perfis de pacientes.

\section{MÉTODOS}

Trata-se de um estudo transversal descritivo, realizado por meio da análise retrospectiva de pacientes submetidos à cirurgia bariátrica aberta pela técnica do bypass gástrico (GBP) em Y de Roux, no Hospital de Clínicas da UFPR, em Curitiba-PR, no período compreendido entre junho de 2013 e dezembro de 2019, analisando 118 pacientes. Foram estudados os dados referentes ao IMC pré e pós-operatório em 6 e 12 meses, e comprimento das alças intestinais no pré-operatório, presentes na descrição cirúrgica, descrita no intraoperatório, antes da confecção do $Y$ de Roux, sendo rotina da equipe avaliar o comprimento do intestino delgado do paciente.

Os resultados obtidos foram quantificados em uma planilha no Google Docs. Os dados estatísticos foram calculados utilizando-se programas próprios para esse fim (SPSS 2.0). Valores de $p$ inferiores a $0,05(p<0,05)$ foram considerados estatisticamente significativos.

Os critérios de inclusão foram pacientes submetidos à cirurgia bariátrica aberta por bypass gástrico em $Y$ de Roux no período entre junho de 2013 e dezembro de 2018, que apresentem prontuários completos e que possuam os valores de IMC pré-operatório e pós-operatório após 6 e 12 meses da cirurgia. Além disso, incluiu os pacientes que apresentaram descrição cirúrgica completa referente aos dados de comprimento do intestino delgado total no intraoperatório.

Os critérios de exclusão foram pacientes menores de 18 anos, presença de prontuários com dados incompletos, ausência de descrição cirúrgica ou ausência de dados em relação ao comprimento das alças do delgado, ausência de acompanhamento regular no pós-operatório, ausência dos níveis de IMC no pré e pós-operatório. Dos 118 pacientes submetidos à cirurgia bariátrica no período estudado, foram excluídos 6 pacientes, sendo que dois deles foram submetidos à Técnica do Sleeve, e 4 prontuários com dados incompletos em relação à perda de peso em 6 e 12 meses ou em relação ao comprimento total do intestino delgado. Dessa forma, foram incluídos 112 pacientes no estudo. O trabalho foi aprovado pelo Comitê de Ética em Pesquisa (CEP) do HC-UFPR pelo protocolo número 36482620.2.0000.0096 (do CAAE).

\section{RESULTADOS}

Dos 112 pacientes submetidos à cirurgia bariátrica pelo by-pass gástrico em $Y$ de Roux considerados no estudo, 93 eram do sexo feminino (83,03\%) e 19 do sexo masculino (16,97\%). A média de idade foi de 41,52 anos, com desvio padrão de 12,22. A média do peso, altura e índice de massa corporal (IMC) foi de $114,3 \mathrm{~kg}, 1,61 \mathrm{~m}$ e $44,08 \mathrm{~kg} / \mathrm{m}^{2}$, respectivamente.

O comprimento médio do intestino delgado total dos pacientes foi de 5,02 metros, sendo que o menor comprimento encontrado foi de 4 metros e o maior, de $6,7 \mathrm{~m}$. Considerandose o sexo feminino, o comprimento médio foi de $5,02 \mathrm{~m}$, e do sexo masculino foi de $5,05 \mathrm{~m}$.

Os resultados acima descritos, bem como o comprimento médio e desvio padrão das alças alimentar, biliopancreática e comum podem ser observados na Tabela 1, representada abaixo.

Em relação ao seguimento dos pacientes no pós-operatório em 6 e 12 meses, verificou-se que a perda de peso média no primeiro semestre, considerando toda a amostra estudada, foi de $27,1 \mathrm{~kg}$, com perda média de 10,4 pontos IMC nesse período. Já no segundo semestre (em 12 meses), a média da perda de peso foi de $35,7 \mathrm{~kg}$ e perda média de 13,8 pontos no IMC. Esses resultados podem ser visualizados na Tabela 2 abaixo.

Considerando-se individualmente os sexos feminino e masculino, ocorreu maior perda de peso em pacientes do sexo masculino, com perda média de $30,9 \mathrm{~kg}$ no seguimento em 6 meses e $40 \mathrm{~kg}$ no seguimento em 12 meses. Por outro lado, nas mulheres, houve perda de peso médio de $26,3 \mathrm{~kg}$ em 6 meses e $34,8 \mathrm{~kg}$ em 12 meses. Entretanto, o peso inicial dos homens era maior (média de 130,9 kg), do que das mulheres (média de 110,9 kg) no pré-operatório. Esses resultados,

Tabela 1 - Perfil da amostra estudada $(n=112)$

\begin{tabular}{|c|c|c|c|}
\hline & $\frac{M}{(n=19)}$ & $\begin{array}{c}F \\
(n=93)\end{array}$ & $\begin{array}{c}\text { Total } \\
(n=112)\end{array}$ \\
\hline Idade & $38,74( \pm 5,55)$ & $42,09( \pm 10,88)$ & $41,52( \pm 12,22)$ \\
\hline Altura & $1,71( \pm 0,06)$ & $1,59( \pm 0,07)$ & $1,61( \pm 0,09)$ \\
\hline Delgado & $5,05( \pm 0,3)$ & $5,02( \pm 0,68)$ & $5,02( \pm 0,74)$ \\
\hline Biliopancreática & $1( \pm 0)$ & $0,99( \pm 0,05)$ & $0,99( \pm 0,05)$ \\
\hline Alimentar & $1( \pm 0)$ & $1,01( \pm 0,05)$ & $1,01( \pm 0,05)$ \\
\hline Alça & $3( \pm 0,3)$ & $3,02( \pm 0,66)$ & $3,02( \pm 0,72)$ \\
\hline Peso & $130,89( \pm 10)$ & $110,91( \pm 16,76)$ & $114,3( \pm 19,52)$ \\
\hline IMC & $44,81( \pm 1,99)$ & $43,93( \pm 5,57)$ & $44,08( \pm 5,91)$ \\
\hline
\end{tabular}

Tabela 2 - Perda de peso e de IMC no seguimento em 6 e 12 meses

\begin{tabular}{|c|c|c|c|c|c|}
\hline Média( $\pm D P$ ) & & Peso & $\begin{array}{c}\text { Perda } \\
\text { de peso }\end{array}$ & IMC & $\begin{array}{l}\text { Perda } \\
\text { de IMC }\end{array}$ \\
\hline & 0 & 114,3 & - & $44,1 \pm$ & - \\
\hline semestres) & 1 & & $27,1( \pm 9,9)$ & $33,6( \pm 5$ & $10,4( \pm 3$ \\
\hline
\end{tabular}


juntamente com a perda de IMC no período estudado, podem ser contemplados na Tabela 3.

Ao realizar correlação entre a perda de peso e o comprimento inicial do intestino delgado, foi possível observar que os pacientes que apresentaram maior perda de peso (maior diferença entre o IMC antes da cirurgia bariátrica e no seguimento em 12 meses) tinham comprimento total de intestino delgado mais longo. Dessa forma, houve uma relação diretamente proporcional entre o comprimento do intestino delgado e a perda de peso, com valor de $p=0,0428(p<0,05)$. A Figura 1 abaixo evidencia esta correlação.

Ao realizar correlação entre o comprimento da alça comum e a perda do IMC (avaliada pela diferença entre o IMC no préoperatório e no seguimento em 12 meses), não houve relação significativamente estatística $(p=0,087-p>0,05)$.

Portanto, não foi possível afirmar nesse estudo que os pacientes que apresentam maior tamanho de intestino delgado na alça comum apresentam maior perda de peso e maior variação no IMC. Essa correlação pode ser evidenciada na Figura 2 abaixo.

Tabela 3 - Perda de peso e IMC no seguimento em 6 e 12 meses, separados por sexo

\begin{tabular}{|c|c|c|c|c|c|}
\hline \multirow[b]{2}{*}{ Média( $\pm \mathrm{DP})$} & \multicolumn{5}{|c|}{ Total (M) } \\
\hline & & Peso & $\begin{array}{c}\text { Perda } \\
\text { de peso }\end{array}$ & IMC & $\begin{array}{l}\text { Perda } \\
\text { de IMC }\end{array}$ \\
\hline \multirow{3}{*}{$\begin{array}{l}\text { Seguimento } \\
\text { (semestres) }\end{array}$} & 0 & $130,9( \pm 10)$ & - & $44,8( \pm 2)$ & - \\
\hline & 1 & $100( \pm 8$ & $30,9( \pm 4,6)$ & $34,3($ & $10,5( \pm 1,4)$ \\
\hline & 2 & & $40( \pm 5,4)$ & $31,3( \pm 1$ & $13,8( \pm 1,7)$ \\
\hline \multirow[b]{2}{*}{ Média( $\pm D P)$} & \multicolumn{5}{|c|}{ Total (F) } \\
\hline & & Peso & $\begin{array}{c}\text { Perda } \\
\text { de peso }\end{array}$ & IMC & $\begin{array}{c}\text { Perda } \\
\text { de IMC }\end{array}$ \\
\hline \multirow{3}{*}{$\begin{array}{l}\text { Seguimento } \\
\text { (semestres) }\end{array}$} & 0 & $110,9( \pm 16,8)$ & - & $43,9( \pm 5,6)$ & - \\
\hline & 1 & $84,4( \pm 14,1)$ & $26,3( \pm 8,7)$ & $33,5( \pm 4,9)$ & $10,4( \pm 3,4)$ \\
\hline & 2 & $74,9( \pm 12,8)$ & $34,8( \pm 10)$ & $29,8( \pm 4,6)$ & $13,8( \pm 3,7)$ \\
\hline
\end{tabular}

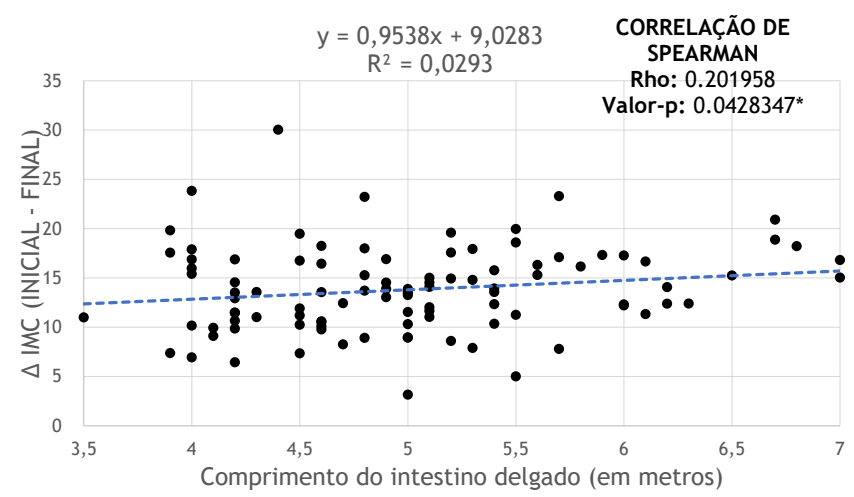

Figura 1 - Relação entre o comprimento do delgado e perda de peso, avaliada pela diferença entre os IMC final e inicial $(p=0,0428)$

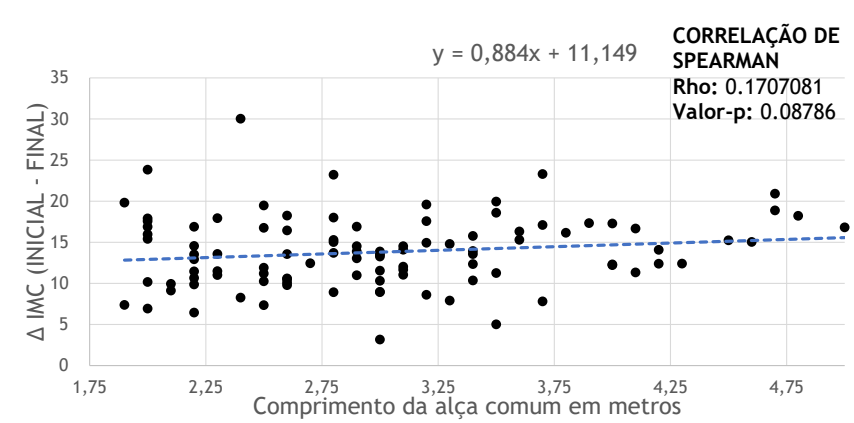

Figura 2 - Relação entre o comprimento da alça comum e a perda de peso, avaliada pela diferença entre os IMC final e inicial $(p=0,08786)$
DISCUSSÃO

O intestino delgado é responsável pela absorção da maioria dos nutrientes e vitaminas necessários ao funcionamento adequado do organismo, como a vitamina D, ferro, ácido fólico, sendo que também apresenta produção hormonal de GLP-1 e peptídeo YY, os quais são essenciais para a saciedade gástrica e o retardo no esvaziamento gástrico, o primeiro também contribuindo para o estímulo à liberação de insulina ${ }^{6}$.

A compreensão do complexo mecanismo de absorção intestinal e a percepção de que os indivíduos apresentam diferentes comprimentos de intestino delgado, desde o ângulo de Treitz até a válvula ileocecal, levaram ao surgimento de diferentes hipóteses relacionadas ao mecanismo da obesidade, propondo que indivíduos que apresentam maior comprimento intestinal apresentam maior absorção nutricional e, por conseguinte, teriam maior tendência à obesidade ${ }^{6}$, conforme a hipótese do intestino longo. O presente estudo mostrou uma relação diretamente proporcional entre o comprimento do intestino delgado total e a perda de peso, com valor de p significativamente estatístico $(p=0,042)$.

Dessa forma, diversos trabalhos foram e estão sendo realizados com o objetivo de compreender melhor os fatores envolvidos na fisiopatologia da obesidade. O comprimento e a função do membro alimentar foram estudados extensivamente, mas poucos estudos estudaram as influências do comprimento do membro biliopancreático ${ }^{9}$.

Sabe-se queo intestino delgado tem seu comprimento variado de 3,36 m a 7,64 m, apresenta diâmetro de aproximadamente $4 \mathrm{~cm}$, representando aproximadamente uma área de $250 \mathrm{~m}^{2}$ (considerando-se pessoa de 1,70 m) para absorção dos nutrientes ${ }^{6}$. Neste estudo foi possível observar um comprimento médio de 5,02 metros ao considerar ambos os sexos. Acredita-se que a perda de peso após desvio biliopancreático e duodenal switch esteja inversamente relacionada ao comprimento do membro alimentar e do canal comum. No entanto, o efeito do comprimento do membro biliopancreático (GLP) na perda de peso tem recebido pouca atenção ${ }^{3}$.

Estudo prévio realizado em 2009 em Hospital Universitário em Curitiba, com 30 pacientes submetidos à gastroplastia pela técnica de Fobi-Capella (RYGB) mostrou que a média do comprimento intestinal para o sexo masculino foi de $582,5 \mathrm{~cm}$ e para o sexo feminino de 509,1 (média de tamanho intestinal de $528,7 \mathrm{~cm}$ para toda a amostra). A partir do cálculo do coeficiente de correlação de Pearson, nesse estudo, confirmouse a ausência de correlação entre IMC e comprimento do intestino delgado ${ }^{6}$. De forma semelhante, o presente estudo mostrou que o comprimento médio total do intestino delgado nas mulheres foi de 5,02 metros e nos homens de 5,05 metros. Em contrapartida, foi possível verificar que houve correlação significativamente estatística entre a perda de peso avaliada pelo IMC e o comprimento total de intestino delgado. Pacientes submetidos à técnica de Fobi-Capella que não apresentaram os resultados esperados, foram propostas outras técnicas para reoperação, com o intuito de atingir maior perda de peso. As opções são as técnicas de Fobi, Brolin ou a gastrojejunoileal distal (tipo Scopinaro). Na primeira é desfeita a êntero-êntero anastomose, a seguir refeita distalmente na metade do comprimento do intestino delgado, que é novamente medido a partir do ângulo de Treitz ${ }^{7}$. Dessa forma, o canal comum e o canal alimentar ficam com 3 a 3,5 m, permanecendo este último com $90 \mathrm{~cm}$. Na técnica tipo Brolin, é desfeita a êntero-êntero anastomose, que é refeita distalmente, a $75 \mathrm{~cm}$ da válvula íleocecal, ficando, então, o canal comum com $75 \mathrm{~cm}$ de comprimento, e além da alça biliopancreática excluída, com $30 \mathrm{~cm}$, o restante do intestino delgado permanece como canal alimentar. Já na técnica de Scorpinaro é desfeita a êntero-êntero anastomose que é refeita com alça aferente jejunal e a alça ileal a $100 \mathrm{~cm}$ 
da válvula ileocecal. Faz-se uma ressecção da parte jejunal da alça alimentar, é refeita uma êntero-êntero anastomose entre o íleo $(220 \mathrm{~cm})$ e o restante da alça jejunal, em média $20 \mathrm{~cm}$, que está anastomosada à bolsa gástrica. Dessa forma, tem-se um canal alimentar composto em média de $20 \mathrm{~cm}$ de jejuno e 220-230 cm de íleo 7 . Em nosso estudo, porém, não foi possível verificar associação estatisticamente significativa entre o comprimento da alça comum e a perda ponderal.

Estudo realizado com 41 doentes, sendo 32 submetidos a reoperação por uma de três técnicas cirúrgicas (Fobi, Brolin e bypass gastrojejunoileal distal) mostrou diminuição da área absortiva do intestino delgado ${ }^{7}$. Dentre essas, a que apresentou resultados superior aos demais em termos de perda de peso foi a técnica de bypass gastrojejunoileal distal (69,7\%). Esse resultado possivelmente ocorreu porque esta técnica aumenta a disaborção e, consequentemente, a perda de peso, porém aumenta a taxa de complicações nutricionais. No presente trabalho, realizamos apenas comparação entre os pacientes submetidos ao by-pass gástrico.

O fator que seguramente apresentou associação positiva entre o comprimento das alças do delgado foi o altura ${ }^{11}$. Os fatores que interferem na perda de peso e na resolução das comorbidades metabólicas são sexo, idade, altura e comprimento jejunal esperado ${ }^{11}$. Assim, a mensuração do comprimento das alças do delgado pode prevenir o risco de consequências nutricionais em procedimentos malabsortivos, revisionais e metabólicos. Nessa análise, para qualquer altura, indivíduos com comprimento jejunal esperado mais longo serão mais obesos e, para qualquer comprimento jejunal esperado, pacientes mais altos terão um peso menor ${ }^{11}$

Estudo com total de 1001 pacientes após desviobiliopancreático/ duodenal switch (209 homens e 792 mulheres, idade média de $42 \pm 10$ anos, índice médio de massa corporal [IMC] $52 \pm$ $9 \mathrm{~kg} / \mathrm{m}$ (2) foi dividido em 2 grupos, de acordo com a razão entre o comprimento do membro biliopancreático (GLP) e o comprimento total do intestino delgado (BLS): um GLP <ou = $45 \%$ do SBL versus um GLP> $45 \%$ do $\mathrm{SBL}^{3}$. Foi percebido que a quantidade de peso perdida após desvio biliopancreático/ duodenal switch está diretamente relacionada à proporção de intestino delgado desviado em pacientes com IMC $>60 \mathrm{~kg} / \mathrm{m}^{2}$. Além disso, o efeito aumentou coma duração do acompanhamento 3 . O comprimento do canal comum também pode ser importante na perda de peso após desvio biliopancreático/duodenal switch, como mostra o estudo de Hamoui et $a l^{3}$, em que pacientes com um canal comum de $100 \mathrm{~cm}$ perderam mais peso do que aqueles com um canal comum de $150 \mathrm{~cm}$. No presente estudo não foi possível realizar essa correlação, pois a padronização da rotina em nosso hospital é realizar alça alimentar e alça biliopancreática com $100 \mathrm{~cm}$ cada uma, e a alça comum é variável de acordo com o comprimento total do delgado do paciente no pré-operatório.

Muitos projetos foram realizados tentando identificar o comprimento ideal do membro alimentar capaz de proporcionar uma perda de peso maior e sustentável com menos comorbidades (principalmente nutricionais), mas até o momento não há consenso ${ }^{12}$. Em uma revisão na literatura com 13 trabalhos, percebeu-se que a liberação de entero-hormônios em resposta a uma carga de alimentos no intestino delgado distal parece desempenhar um papel importante na remissão de comorbidades. Portanto, o comprimento da alça biliopancreática pode afetar esse processo ${ }^{12}$. A restrição gástrica combinada com um grau modesto de desvio da alça biliopancreática resultou em uma perda de peso pós-operatória significativamente maior em comparação com o by-pass em $Y$ de Roux convencional, com comprimentos de alça de $15 \mathrm{~cm}$ para a alça biliopancreática e $75 \mathrm{~cm}$ para a alça alimentar ${ }^{12}$.

Além da maior perda de peso, o aumento do comprimento da alça biliopancrática poderia estar relacionado com a melhora metabólica do diabetes. A maior série até o momento comparando comprimentos diferentes de alça com mais de 500 pacientes não encontrou diferença na $\mathrm{HbA} 1 \mathrm{c}$ em pacientes diabéticos entre uma alça longa $(200 \mathrm{~cm})$ e um RYGB clássico; no entanto, o RYGB da alça biliopancreática longa foi associada a uma diminuição significativa da $\mathrm{HbA} 1 \mathrm{c}$ em comparação ao GBP clássico em pacientes não diabéticos ${ }^{12}$.

É conhecido que, em pacientes super-obesos, as taxas de perda de peso e recuperação de peso são altas após o bypass em $Y$ de Roux, sendo que outro estudo mostrou que para melhorar a perda de peso, é necessário o alongamento do membro biliopancreático ${ }^{10}$. Foi realizada uma coorte retrospectiva com 671 pacientes super-obesos operados em um período de 10 anos. Os pacientes foram classificados em três grupos: (1) 155 pacientes; alça comum $150 \mathrm{~cm}$, alça biliopancreática $60 \mathrm{~cm}$; (2) 230 pacientes; alça comum $60 \mathrm{~cm}$, alça biliopancreática 200 cm; e (3) 286 pacientes; alça comum $150 \mathrm{~cm}$, alça biliopancreática $200 \mathrm{~cm}$. O comprimento total da alça biliopancrática foi encurtado com $60 \mathrm{~cm}$ no grupo 1 e com $200 \mathrm{~cm}$ nos grupos 2 e 3 . Ao comparar os três grupos, percebeu-se que a falha da perda de peso foi maior no grupo 1 (10,3\%) em relação aos demais grupos (4,3\%; 5,2\%). O grupo 3 teve recuperação de peso significativamente menor (26,6\%). A remissão de comorbidades foi maior nos grupos com 2 metros de alça biliopancreática em detrimento das deficiências nutricionais e vitamínicas (3,9\%; 5,9\%). Não foi observada diferença na hipoalbuminemia ${ }^{10}$.

Considerando que a perda de peso foi semelhante nos grupos com $200 \mathrm{~cm}$ de comprimento, outro estudo mostrou que o tamanho da alça alimentar é de extrema importância para a perda de peso a longo prazo, em vez de focar principalmente no encurtamento do canal comum ${ }^{10}$. Dessa forma, foi proposto encurtar a alça alimentar, com uma alça biliopancreática de $200 \mathrm{~cm}$ e uma alça comum de $100 \mathrm{~cm}$. Tendo em vista que o comprimento total do intestino delgado é variável entre as pessoas, o trabalho sugere que reduzir o comprimento da alça alimentar em um terço - em vez de um número fixo - é particularmente importante em casos de comprimento muito curto do intestino delgado, a fim de evitar desnutrição e má absorção ${ }^{10}$. Neste estudo não foi encontrado relação estatisticamente significativa $(p>0,05)$ entre o tamanho da alça comum e a perda de peso. A comparação entre o tamanho da alça alimentar e a perda de peso não foi realizada, pois a alça alimentar apresenta comprimento padronizado em torno de $100 \mathrm{~cm}$ na rotina do nosso serviço, conforme anteriormente citado.

Amaioria dos estudos publicados não levamem consideração a influência da alça comum na perda de peso, apenas das alças alimentar e biliopancreática. $O$ estudo realizado na Espanha ${ }^{1}$ com 151 pacientes submetidos à cirurgia bariátrica videolaparoscópica por by-pass em Y de Roux (RYGB) mostrou que a alça comum não tem efeito na perda de peso em pacientes com RYGB, e que um comprimento reduzido na alça comum está relacionado a maiores deficiências nutricionais. Os pacientes foram divididos em 2 grupos de acordo com o índice de massa corporal (IMC): (1) obesos mórbidos (IMC 35-50 kg / m2) com 115 pacientes; e (2) pacientes com obesidade mórbida e obesidade (IMC > $50 \mathrm{~kg} / \mathrm{m} 2$ ) com 36 pacientes. O comprimento do membro biliopancreático foi de $100 \mathrm{~cm}$ nos dois grupos; o membro alimentar foi de $150 \mathrm{~cm}$ no grupo de obesos (primeiro grupo) e de $200 \mathrm{~cm}$ no grupo de super-obesos (segundo grupo). A porcentagem de alça comum de $50 \%$ está associada estatisticamente a deficiências de ferro, ferritina e proteína (proteína total e albumina). Portanto, exames de sangue nutricionais mais rigorosos devem ser realizados para oferecer tratamento precoce com suplementos e fornecer uma abordagem correta ao paciente ${ }^{1}$. Entretanto, considerando que a porcentagem de intestino 
utilizada no membro comum não influencia a porcentagem de excesso de perda de peso em pacientes obesos ou super obesos que recebem RYGB laparoscópico, não há indicação em alterar o comprimento da alça comum visando a perda de peso. De forma semelhante, nesta casuísica também não foi possível realizar uma associação estatística significante entre o comprimento da alça comum e a perda de peso $(p=0,087-p>0,05)$.

Em relação à variação do comprimento das alças alimentar e biliopancreática e a melhora dos parâmetros metabólicos (DM, HAS, dislipidemia e circunferência abdominal), estudos mostram que não houve relação entre as variáveis ${ }^{8}$. Esse estudo foi realizado com 63 pacientes, em coorte retrospectivo, e foram divididos em três grupos: grupo 1: alça biliopancreática de $50 \mathrm{~cm}$ e alça alimentar de 100 cm; grupo 2: alça biliopancreática de $50 \mathrm{~cm}$ e alça alimentar de $150 \mathrm{~cm}$ e grupo 3: alça biliopancreática de $100 \mathrm{~cm}$ e alça alimentar de $150 \mathrm{~cm}$. Ao comparar os grupos, também não houve diferença estatística na porcentagem de perda do excesso de peso entre os grupos e as medidas da cintura abdominal reduziram de forma homogênea em todos os grupos ${ }^{8}$.

Comparando grupos com diferentes comprimentos de alça alimentare biliopancreática, outros autores demonstraram diferença no controle do DM2 analisando dois grupos com alças de extensões diferentes (grupo 1: alça biliopancreática de $50 \mathrm{~cm}$ e alimentar de $150 \mathrm{~cm}$; grupo 2: alça biliopancreática de $100 \mathrm{~cm}$ e alimentar de $250 \mathrm{~cm})^{8}$. O diabetes foi controlado em $58 \%$ do grupo 1 e em $93 \%$ do grupo $2(P<0,05)$. Os distúrbios lipídicos melhoraram em $57 \%$ do grupo 1 e em $70 \%$ do grupo $2(P<0,05)$. Nenhuma diferença estatística foi encontrada no controle ou melhora da hipertensão, apneia do sono ou distúrbio do refluxo gastroesofágico. A perda de excesso de peso foi mais rápida no grupo 1 , mas foi semelhante nos dois grupos aos 48 meses (70\% no grupo 1 e $74 \%$ no grupo 2 ), sem diferença estatística. Os com desvios intestinais mais longos tiveram melhor controle do DM2 $(p<0,05)^{8}$.

$A$ insucesso da cirurgia bypass gástrico em $Y$ de Roux (RYGB) pode ser definido como falha na perda de peso, definido como perda de excesso de peso (EWL) $<50 \%$ ou índice de massa corporal $(\mathrm{IMC})>35 \mathrm{~kg} / \mathrm{m}^{2}$, e o ganho de peso ocorre em até $35 \%$ dos pacientes ${ }^{4}$. Não existe consenso em relação à melhor técnica para revisão da cirurgia inicial. Uma das opções é a conversão do RYGB para uma alça biliopancreático longa (BPL) e os resultados a curto prazo são promissores. Pesquisa realizada com 28 pacientes submetidos à cirurgia revisional, com comprimentos de $150 \mathrm{~cm}$ (IC95\% 133-156 cm) para a alça biliopancreática e $100 \mathrm{~cm}$ (IC $95 \% 97-113 \mathrm{~cm}$ ) para a alça alimentar, fornecendo assim um comprimento mediano total de $250 \mathrm{~cm}^{4}$. O principal princípio técnico para obter maior perda de peso foi o encurtamento do comprimento total do membro alimentar (e a exclusão de uma maior quantidade de intestino delgado na alça biliopancreática $)^{4}$. Essa cirurgia resultou em uma redução adicional do IMC de $10,0 \mathrm{~kg} / \mathrm{m}^{2}$, no entanto, a alta taxa de desnutrição proteica calórica com comprimento comum de $250 \mathrm{~cm}$ suscita grandes preocupações em relação à praticabilidade geral e segurança dessa técnica revisional malabsorptiva, sendo que seis pacientes precisaram ser reoperados devido à desnutrição grave dessa técnica ${ }^{4}$. Sendo assim, não se recomenda realizar redução no comprimento da alça alimentar em pacientes com risco de desnutrição, motivo pelo qual mantivemos o padrão de $100 \mathrm{~cm}$ desta alça em nosso Serviço.

Finalmente, a literatura mostra uma ampla gama de variáveis relacionadas à perda de peso nos pacientes submetidos à cirurgia bariátrica, tais como a técnica utilizada, o comprimento das alças no bypass gástrico em $Y$ de Roux e também a rotina de acompanhamento nutricional e físico do paciente

\section{CONCLUSÃO}

Concluindo, foi possível verificar uma relação diretamente proporcional entre o comprimento do intestino delgado e a perda de peso, porém não foi obtida correlação estatisticamente significativa entre o comprimento da alça comum e a perda de peso, evidenciando que possivelmente apenas o comprimento das alças alimentar e biliopancreática apresentem influência na perda de peso, conforme sugerem outros estudos. Portanto, é importante avaliar não apenas detalhes técnicos do procedimento cirúrgico, mas verificar a perda de peso avaliando-se o paciente como um todo e as outras variáveis presentes.

\section{REFERÊNCIAS}

1. Abellan I, Luján J, Frutos MD, Abrisqueta J, Hernández Q, López V, Parrilla P. The influence of the percentage of the common limb in weight loss and nutritional alterations after laparoscopic gastric bypass. Surg Obes Relat Dis. 2014;10(5):829-33. doi: 10.1016/j. soard.2014.06.009.

2. DeOliveira GJM, SchieferdeckerMEM,Campos ACL. Are enterotypes in obese modified by bariatric surgery, the use of probiotic supplements and food habits? Arq Bras Cir Dig. 2021;34(2):e1601. doi: 10.1590/0102-672020210002e1601.

3. Hamoui N, Anthone GJ, Kaufman HS, Crookes PF. Maintenance of weight loss in patients with body mass index $>60 \mathrm{~kg} / \mathrm{m} 2$ : importance of length of small bowel bypassed. Surg Obes Relat Dis. 2008;4(3):404-6; discussion 406-7.doi:10.1016/j.soard.2007.08.020.

4. Kraljević M, Köstler T, Süsstrunk J, Lazaridis II, Taheri A, Zingg U, Delko T. Revisional Surgery for Insufficient Loss or Regain of Weight After Roux-en-YGastric Bypass: Biliopancreatic Limb Length Matters. Obes Surg. 2020;30(3):804-811. doi: 10.1007/s11695-019-04348-8.

5. Lucas RWDC, Nassif PAN, Tabushi FI, Nassif DSB, Ariede BL, BritesNeto J, Malafaia O. Can stature, abdominal perimeter and BMl index predict possible cardiometabolic risks in future obesity? Arq Bras CirDig.2020;33(2):e1529. doi: 10.1590/0102-672020200002e1529.

6. NassifPAN, MalafaiaO, RibasCAPM, PachnickiJPA, KumeMH, Macedo L et al . Correlation study of BMl and small intestine length in obese patientssubjected to bariatricsurgery.ArqBrasCirDig.2009;22(3):153157. https://doi.org/10.1590/S0102-67202009000300004.

7. Pareja JC, Pilla VF, Callejas-Neto F, Coelho-Neto Jde S, Chaim EA, Magro DO. Gastric bypass Roux-en-Y gastrojejunostomy-conversion to distal gastrojejunoileostomy for weight loss failure - experience in 41 patients. Arq Gastroenterol. 2005;42(4):196-200. doi: 10.1590/s0004-28032005000400002.

8. Pinheiro JS, Schiavon CA, Pereira PB, Correa JL, Noujaim P, Cohen R. Long-long limb Roux-en-Y gastric bypass is more efficacious in treatment of type 2 diabetes and lipid disorders in super-obese patients. Surg Obes Relat Dis. 2008;4(4):521-5; discussion 526-7. doi: 10.1016/j.soard.2007.12.016.

9. Ramos RJ, Mottin CC, Alves LB, Benzano D, Padoin AV. Effect of size of intestinal diversions in obese patients with metabolic syndrome submitted to gastric bypass. Arq Bras Cir Dig. 2016;(Suppl 1):1519. doi: 10.1590/0102-6720201600S10005.

10. Shah K, Nergård BJ, Fagerland MW, Gislason H. Limb Length in Gastric Bypass in Super-Obese Patients-Importance of Length of Total Alimentary Small Bowel Tract. Obes Surg. 2019;29(7):20122021. doi: 10.1007/s11695-019-03836-1.

11. Tacchino RM. Bowel length: measurement, predictors, and impact on bariatric and metabolic surgery. Surg Obes Relat Dis. 2015;11(2):328-34. doi: 10.1016/j.soard.2014.09.016.

12. Zorrilla-Nunez LF, Campbell A, Giambartolomei G, Lo Menzo E, Szomstein S, Rosenthal RJ. The importance of the biliopancreatic limb length in gastric bypass: A systematic review. Surg Obes Relat Dis. 2019;15(1):43-49. doi: 10.1016/j.soard.2018.10.013. 